\title{
Evidencia serológica de leptospirosis canina en la comunidad indígena Kamentsá, Putumayo, Colombia
}

\author{
SEROLOGICAL EVIDENCE OF CANINE LEPTOSPIROSIS IN THE KAMENTSÁ INDIGENOUS \\ community, Putumayo, Colombia \\ Marlyn H. Romero ${ }^{1,3}$, Myriam Astudillo², Disney M. Aguillón', Ileany D. Lucio ${ }^{1}$
}

\section{Resumen}

El objetivo del estudio fue determinar la prevalencia de leptospirosis canina e identificar factores asociados con la infección en una población indígena Kamentsá del departamento de Putumayo, Colombia. Se evaluaron 154 caninos de ambos sexos localizados en el área rural del Valle de Sibundoy. Se identificaron variables demográficas, sanitarias y de tenencia responsable mediante una encuesta estructurada. Se colectaron muestras de sangre a los canes y se procesaron mediante la técnica de microaglutinación macroscópica (MAT) usando un cepario de referencia conformado por 24 serogrupos de Leptospira. La prevalencia de anticuerpos contra Leptospira sp fue de 79.9\%. Los serogrupos más frecuentes fueron Gripotyphosa (10.2\%), Hursbridge (8.4\%), Sarmin y Australis (7.2\%), Canicola (6.8\%) y Tarassovi (6.4\%). Los factores de riesgo asociados fueron la edad del canino, la presencia de aguas servidas y la tenencia de más de un canino en la vivienda $(\mathrm{p}<0.05)$. En conclusión, la alta prevalencia de anticuerpos contra Leptospira sp en los caninos sugiere que existe un riesgo potencial de transmisión de la bacteria al hombre y, por tanto, es necesario fortalecer las medidas de promoción, prevención y vigilancia por parte de las autoridades de Salud Pública.

Palabras clave: epidemiología; leptospiras; perros; serogrupos

\section{Abstract}

The objective of this study was to determine the prevalence of canine leptospirosis and identify factors associated with the infection in an indigenous Kamentsá population of the department of Putumayo, Colombia. Blood samples of dogs of both sexes $(n=154)$

\footnotetext{
${ }^{1}$ Departamento de Salud Animal, Facultad de Ciencias Agropecuarias, Universidad de Caldas, Manizales, Colombia

${ }^{2}$ Departamento de Microbiología, Universidad del Valle, Cali, Valle, Colombia

${ }^{3}$ E-mail: marlyn.romero@ucaldas.edu.co
} 
located in the rural area of Sibundoy Valley were collected. Demographic, sanitary, and responsible tenure variables were identified through a structured survey. Blood samples were processed by the macroscopic microagglutination test (MAT) using a reference strain collection composed of 24 serogroups of Leptospira serogroups. The prevalence of antibodies against Leptospira sp was $79.9 \%$. The most frequent serogroups were Gripotyphosa (10.2\%), Hursbridge (8.4\%), Sarmin and Australis (7.2\%), Canicola (6.8\%) and Tarassovi (6.4\%). The associated risk factors were the age of the dog, the presence of sewage and the possession of more than one dog in the house $(\mathrm{p}<0.05)$. In conclusion, the high prevalence of antibodies against Leptospira sp in dogs suggests that there is a potential risk of transmission of the bacteria to man and, therefore, it is necessary to strengthen the promotion, prevention and surveillance measures by Public Health authorities.

Key words: epidemiology; leptospiras; dogs; serogroups

\section{INTRODUCCIÓN}

La leptospirosis es una enfermedad sistémica causada por bacterias patógenas del género Leptospira que afecta a humanos, mamíferos silvestres y domésticos, de zonas urbanas y rurales (Ward y Kelman, 2011) y se considera una zoonosis de distribución mundial a excepción de la Antártida (Adler y de la Peña Moctezuma, 2010).

La fuente de infección de las leptospiras en humanos es el contacto directo o indirecto con la orina de animales infectados (Levett, 2004). Los caninos juegan un rol importante en la epidemiología de la leptospirosis humana como reservorios y fuentes de infección, por su estrecho vínculo con el hombre y su constante interacción en espacios comunes, aspectos que aumentan la probabilidad de infección entre especies (Romero et al., 2012). Así mismo, los caninos pueden presentar la condición de portador durante años, al mantener las leptospiras viables y con capacidad de multiplicarse en sus riñones y excretarlas de manera intermitente por la orina (Levett, 2004).

En Colombia, el reporte de los casos y muertes por leptospirosis entre humanos se hace al Instituto Nacional de Salud, mediante el Sistema de Vigilancia en Salud Pública
(SIVIGILA) (Yusti et al., 2013). No obstante, existe poca evidencia de la situación epidemiológica de la leptospirosis en poblaciones indígenas, debido al bajo acceso a los servicios de salud pública, la imposibilidad de contar con laboratorios de diagnóstico locales y a factores culturales que favorecen el tratamiento de síndromes febriles a través de la medicina no tradicional, entre otros, lo cual conlleva a un subregistro de los eventos en salud (Ortiz et al., 2015).

En la comunidad indígena Kamentsá del Putumayo se desconoce la presencia de la infección en los caninos, los cuales, junto con los roedores sinantrópicos, son considerados como el principal enlace para la diseminación de las leptospiras en áreas urbanas y rurales (Oliveira-Lavinsky et al., 2012). El objetivo del estudio fue determinar la prevalencia de anticuerpos contra Leptospira sp en caninos e identificar factores asociados en la población indígena Kamentsá.

\section{Materiales y Métodos}

El estudio contó con la aprobación del Comité de Ética de la Universidad de Caldas. Así mismo, se siguieron los lineamientos en cuanto a las normas éticas para el manejo de animales de laboratorio, además de los 
expuestos en la Ley 84 de 1989 y el Decreto 2257 de 1986. Se coordinó con el gobernador de la comunidad indígena y se obtuvo el consentimiento informado de los propietarios de los animales, previo a la toma de las muestras sanguíneas.

\section{Área de Estudio}

Se realizó un estudio descriptivo transversal en junio y julio de 2014 en el territorio habitado por la comunidad indígena Kamentsá, en el Valle de Sibundoy (Putumayo) (108'57'N 76³8'47'O), localizado a $2200 \mathrm{msnm}$. El área del valle es de $525 \mathrm{~km}^{2}$, la temperatura promedio de $16{ }^{\circ} \mathrm{C}$ y humedad relativa de $86 \%$, con una precipitación anual de $1432 \mathrm{~mm}$ (IDEAM, Colombia). La comunidad está conformada por 4879 personas según la Organización de las Naciones Unidas (ONU).

\section{Factores de Riesgo}

Se evaluaron variables demográficas, sanitarias y de tenencia responsable de las mascotas mediante la aplicación de una encuesta estructurada dirigida a los propietarios de los caninos.

\section{Muestras y Análisis de Laboratorio}

Se hizo un muestreo por conveniencia de 154 caninos criollos del área rural, de las veredas Machindoy $(n=6)$, Tabanoka $(n=13)$, Sotanjoy ( $\mathrm{n}=9$ ), El Egido ( $\mathrm{n}=11)$, Cabuyayaco $(n=15)$, Sagrado Corazón $(n=27)$, Leandro Agreda $(n=17)$, Fátima Carrizallaco $(n=20)$, Alto Resguardo $(n=7)$ y Tamabioy $(n=29)$. El criterio de exclusión fue la vacunación previa contra leptospirosis. Las muestras de sangre se obtuvieron por punción de la vena cefálica $(4 \mathrm{ml})$, se centrifugaron y los sueros resultantes se almacenaron a $-20{ }^{\circ} \mathrm{C}$ hasta el momento de su análisis.

El procesamiento de las muestras se efectuó en el laboratorio de diagnóstico de leptospirosis de la Universidad del Valle, Cali. El mantenimiento de las cepas y el manejo de la técnica de microaglutinación (MAT) se realizó bajo los parámetros convencionales (Astudillo et al., 2009), utilizando un cepario de referencia suministrado por el laboratorio internacional de referencia para el diagnóstico de la leptospirosis del Royal Tropical Institute (Amsterdam, Holanda). Los serogrupos evaluados fueron: Bataviae, Mini, Autumnalis, Canicola, Shermani, Icterohaemorrhagiae, Cynopteri, Australis, Celledoni, Grippotyphosa, Javanica, Manhao, Pomona, Pyrogenes, Sejröe, Tarassovi, Ballum, Louisiana, Djasiman, Semaranga, Hursbridge, Ranarum, Panama y Sarmin. Se consideraron como positivos los sueros con títulos $\geq 1: 100$ (Romero et al., 2009).

\section{Análisis Estadístico}

Se realizó un análisis de regresión logística multivariada que asumió como variable de respuesta binomial los resultados de la prueba MAT, donde 0 correspondió a los caninos con resultados negativos y 1 a los animales seropositivos. Se realizó la prueba de bondad de ajuste del modelo obtenido por medio del estadístico de Hosmer y Lemeshow. Los efectos de las variables predictoras sobre el resultado positivo o negativo de la prueba se expresaron por medio de las razones de disparidad (OR) y sus respectivos intervalos de confianza del 95\%. Los valores de $\mathrm{p}<0.05$ fueron considerados como significativos. El análisis se efectuó utilizando el programa Stata v. 13.0 (College Station, Texas, EEUU).

\section{Resultados}

La seroprevalencia de la infección por Leptospira sp en caninos fue de 79.9\% (123/ 154). Los sueros reaccionaron con la totalidad de los serogrupos incluidos en MAT y los más frecuentes fueron Gripotyphosa (10.2\%), Hursbridge (8.4\%), Sarmin y Australis (7.2\%), Canicola (6.8\%) y Tarassovi (6.4\%) (Cuadro 1). El rango de los títulos de anticuerpos obtenido por la prueba MAT fue 
Cuadro 1. Distribución de los sueros de caninos positivos a leptospirosis con la prueba de microaglutinación (MAT) de acuerdo con los serogrupos reaccionantes y el título (UI) alcanzado (Kamentsá, Valle de Sibundoy, Putumayo)

\begin{tabular}{|c|c|c|c|c|c|c|c|c|c|}
\hline \multirow{2}{*}{ Serovar } & \multicolumn{7}{|c|}{ Caninos seropositivos } & \multicolumn{2}{|c|}{ Total } \\
\hline & $1 / 50$ & $1 / 100$ & $1 / 200$ & $1 / 400$ & $1 / 800$ & $1 / 1600$ & $>1 / 1600$ & $\mathrm{n}$ & $\%$ \\
\hline Australis & 19 & 11 & 4 & 2 & & & & 36 & 7.2 \\
\hline Automnalis & 11 & 3 & 1 & & & & & 15 & 3.0 \\
\hline Ballum & 19 & 8 & 3 & 1 & & & & 31 & 6.2 \\
\hline Bataviae & 3 & 3 & 1 & & 1 & 1 & & 9 & 1.8 \\
\hline Canicola & 17 & 10 & 4 & 2 & 1 & & & 34 & 6.8 \\
\hline Celledoni & 2 & 1 & & & & & & 3 & 0.6 \\
\hline Cynopteri & 5 & 4 & 1 & & & & & 10 & 2.0 \\
\hline Djasiman & 10 & 3 & 3 & 1 & & & & 17 & 3.4 \\
\hline Grippotyphosa & 32 & 13 & 5 & 1 & & & & 51 & 10.2 \\
\hline Hursbridge & 21 & 11 & 6 & 4 & & & & 42 & 8.4 \\
\hline Icterohaemorragie & 6 & 2 & 4 & 1 & & 1 & & 14 & 2.8 \\
\hline Javanica & 8 & 5 & 4 & & & & 1 & 18 & 3.6 \\
\hline Louisiana & 6 & 1 & 3 & & & & & 10 & 2.0 \\
\hline Manhao & 3 & 4 & 1 & 3 & & 1 & & 12 & 2.4 \\
\hline Mini & 11 & 7 & 5 & 3 & 1 & & & 27 & 5.4 \\
\hline Panama & 17 & 8 & 1 & 1 & & 2 & & 29 & 5.8 \\
\hline Pomona & 4 & 4 & 2 & & & & & 10 & 2.0 \\
\hline Pyrogenes & 10 & 5 & 3 & 2 & & & & 20 & 4.0 \\
\hline Ranarum & 6 & 4 & & & & & & 10 & 2.0 \\
\hline Sarmin & 16 & 6 & 4 & 7 & 1 & 1 & 1 & 36 & 7.2 \\
\hline Sejroe & 5 & 5 & 5 & & & & & 15 & 3.0 \\
\hline Semaranga & 9 & 1 & & & & & & 10 & 2.0 \\
\hline Shermani & 6 & 1 & & & & & 1 & 8 & 1.6 \\
\hline Tarassovi & 22 & 7 & 2 & & 1 & & & 32 & 6.4 \\
\hline Total (n) & 268 & 127 & 62 & 28 & 5 & 6 & 3 & 499 & \\
\hline$(\%)$ & 53.7 & 25.5 & 12.4 & 5.6 & 1.0 & 1.2 & 0.6 & & 100 \\
\hline
\end{tabular}

de 1:100 hasta 1:1600. Se observaron coaglutinaciones en el $36.5 \%$ de los sueros positivos. Los títulos más altos de MAT se obtuvieron contra los serogrupos Javanica, Sharmani y Sarmin.

Se presentaron casos positivos a la infección en todas las veredas de la comunidad Kamentsá en un rango entre 44.4 y $100 \%$. La comunidad se dedicaba a la producción de aves y cuyes (79.2\%) y de bovinos y porcinos (7.1\%). El 63\% (97/154) era propietario de más de un canino. De otra parte, el $40.9 \%(n=63)$ de las viviendas estaba habitada por 1 a 5 personas, el $52.6 \%(\mathrm{n}=81)$ entre 6 y 10 y el $6.5 \%$ entre 11 y 20 . El $84.1 \%$ $(n=145)$ de los caninos era alimentado con sobras de alimentos, el $28.9 \%$ tenía un plan sanitario de vermifugación ( $\mathrm{n}=24$ ) y el $28.6 \%$ $(n=23)$ fue vacunado contra la rabia. Otras variables que se consideraron como potenciales factores de riesgo para la transmisión 
Cuadro 2. Factores potencialmente asociados con la presencia de anticuerpos anti-Leptospira sp en 154 caninos de la comunidad indígena Kamentsá (Valle de Sibundoy, Putumayo)

\begin{tabular}{|c|c|c|c|c|c|c|}
\hline \multirow{2}{*}{ Variable } & \multicolumn{3}{|c|}{ Positivo } & \multicolumn{2}{|c|}{ Negativo } & \multirow[t]{2}{*}{$\mathrm{p}$} \\
\hline & N. ${ }^{\circ}$ & $\mathrm{n}$ & $\%$ & $\mathrm{n}$ & $\%$ & \\
\hline $\begin{array}{l}\text { Edad del can } \\
\text { (años) }\end{array}$ & & & & & & 0.02 \\
\hline$<1$ & 28 & 17 & 13.82 & 11 & 35.5 & \\
\hline $1-4$ & 93 & 76 & 61.8 & 17 & 54.8 & \\
\hline $5-8$ & 24 & 22 & 17.9 & 2 & 6.5 & \\
\hline$>9$ & 9 & 8 & 6.5 & 1 & 3.2 & \\
\hline Sexo del can & & & & & & $\mathrm{ns}$ \\
\hline Machos & 99 & 76 & 61.8 & 23 & 74.2 & \\
\hline Hembras & 55 & 47 & 38.2 & 8 & 25.8 & \\
\hline $\begin{array}{l}\text { Manejo aguas } \\
\text { servidas }\end{array}$ & & & & & & 0.03 \\
\hline Sí & 90 & 67 & 54.5 & 23 & 74.2 & \\
\hline No & 64 & 56 & 45.5 & 8 & 25.8 & \\
\hline Densidad $>1$ can & & & & & & 0.04 \\
\hline Sí & 122 & 94 & 76.4 & 28 & 90.3 & \\
\hline No & 32 & 29 & 23.6 & 3 & 9.7 & \\
\hline $\begin{array}{l}\text { Bovinos y } \\
\text { porcinos }\end{array}$ & & & & & & ns \\
\hline Sí & 11 & 9 & 7.3 & 2 & 6.5 & \\
\hline No & 143 & 114 & 92.7 & 29 & 93.5 & \\
\hline $\begin{array}{l}\text { Recolección de } \\
\text { residuos sólidos }\end{array}$ & & & & & & ns \\
\hline Sí & 49 & 37 & 30.08 & 12 & 38.7 & \\
\hline No & 105 & 86 & 69.9 & 19 & 61.3 & \\
\hline $\begin{array}{l}\text { Permanencia en } \\
\text { la vivienda }\end{array}$ & & & & & & ns \\
\hline Sí & 140 & 112 & 91.0 & 28 & 90.3 & \\
\hline No & 14 & 11 & 8.9 & 3 & 9.7 & \\
\hline Roedores & & & & & & $\mathrm{ns}$ \\
\hline Sí & 90 & 71 & 57.7 & 19 & 61.3 & \\
\hline No & 64 & 52 & 42.3 & 12 & 38.7 & \\
\hline
\end{tabular}

N. ${ }^{\circ}$ : número total de caninos; ns: no signficativo

de la leptospirosis canina se presentan en el Cuadro 2.

De acuerdo con el análisis de regresión logística multivariada, los caninos con edades comprendidas entre los 5 y 8 años tuvieron 7.7 veces más riesgo de ser seroreactivos a MAT al compararse con los caninos menores de un año $(O R>1 ; p<0.05)$. Tener una baja densidad de caninos en la vivienda y contar con tratamiento de aguas servidas se consideraron como factores protectores $(\mathrm{OR}<1)$ (Cuadro 3). 
Cuadro 3. Factores asociados con las tasas de incidencia de leptospirosis canina en la comunidad indígena Kamentsá (Valle de Sibundoy, Putumayo) por medio de un modelo de regresión logística multivariada

\begin{tabular}{llllc}
\hline Variable/categoría & OR & EE & IC 95\% & P \\
\hline Edad (años) & Referencia & & & \\
$\quad<1$ & 2.5 & 0.07 & $0.9-3.3$ & 0.04 \\
$1-4$ & 7.7 & 0.05 & $1.3-7.9$ & 0.01 \\
$5-8$ & 5.3 & 0.05 & $3.3-6.7$ & $\mathrm{~ns}$ \\
$>9$ & & & \\
$\begin{array}{l}\text { Densidad (>1 can en la } \\
\text { vivienda) }\end{array}$ & Referencia & & & \\
$\quad$ Sí & 0.26 & 0.05 & $0.61-0.8$ & 0.04 \\
$\quad$ No & & & & \\
Tratamiento de aguas & & & & \\
servidas & Referencia & & & \\
$\quad$ Sí & 0.4 & 0.08 & $0.15-0.9$ & 0.03 \\
No &
\end{tabular}

OR: razón de disparidad; ns: no significativo; EE: error estándar; IC: intervalo de confianza

\section{Discusión}

La seroprevalencia de $79.9 \%$ hallada en el presente estudio es la más alta reportada en población canina urbana y rural, así como en animales callejeros y localizados en barrios marginales de Colombia (Cuadro 4). Por otro lado, el registro de casos de leptospirosis humana en el departamento de Putumayo fue bajo, reporte que correspondió a 4, 1, 5 y 3 casos en 2012, 2013, 2014 y 2015, respectivamente (SIVIGILA, 2014). Estos hallazgos son desconcertantes y sugieren que existe un subregistro de la enfermedad, tanto en humanos como en caninos y otras especies animales, lo cual puede conllevar a un problema de salud pública grave. Se tiene que considerar que la mayor proporción de los propietarios de los animales encuestados moraban en viviendas compartidas entre 6 y 20 personas, aspecto que aumenta el riesgo de transmisión de la enfermedad (Lelu et al., 2015), junto con otros factores, tales como deficiencias en el saneamiento básico por la falta de tratamiento de las aguas servidas y de los residuos sólidos, así como una baja cobertura de inmunización canina.

De otra parte, los caninos evaluados no habían sido vacunados contra leptospirosis, por lo que la reactividad de los sueros a un pool de 24 serogrupos no se puede interpretar como una aglutinación cruzada entre estos, sino al posible contacto con varias especies de animales domésticos y silvestres que actúan como reservorios de leptospiras (Lelu et al., 2015), ya que la transmisión directa de la infección entre caninos es infrecuente (Ward, 2002). Así mismo, las leptospiras no exhiben la misma virulencia y su asociación con los casos clínicos de la enfermedad es compleja, aspecto que dificulta la interpretación de los resultados (André-Fontaine, 2006). Por ejemplo, los serogrupos Grippotyphosa y Australis fueron aglutinados con frecuencia; no obstante, esta última ha sido responsable de más casos de leptospirosis subaguda y crónica que la primera (AndréFontaine, 2006). 
Cuadro 4. Estudios de prevalencia de leptospirosis canina en Colombia (2004-2013)

\begin{tabular}{|c|c|c|c|c|c|c|}
\hline Departamento & Prueba & $\begin{array}{l}\text { Prevalencia } \\
\quad(\%)\end{array}$ & Serogrupos & N. ${ }^{\circ}$ & Área & Autor \\
\hline $\begin{array}{l}\text { Valle del } \\
\text { Cauca }\end{array}$ & MAT & 1.1 & $\begin{array}{l}\text { Icterohaemorrhagiae } \\
\text { Hardjo } \\
\text { Gryppotyphosa } \\
\text { Canicola }\end{array}$ & 197 & $\begin{array}{l}\text { Urbana } \\
\text { Callejeros }\end{array}$ & $\begin{array}{l}\text { (Rodríguez } \\
\text { et al., } \\
\text { 2004) }\end{array}$ \\
\hline Boyacá & MAT & 29.0 & $\begin{array}{l}\text { Canicola } \\
\text { Gryppotyphosa }\end{array}$ & 51 & $\begin{array}{l}\text { Urbana } \\
\text { Callejeros }\end{array}$ & $\begin{array}{l}\text { (Astudillo } \\
\text { et al., } \\
\text { 2009) }\end{array}$ \\
\hline $\begin{array}{l}\text { Valle del } \\
\text { Cauca }\end{array}$ & MAT & 31.0 & $\begin{array}{l}\text { Icterohaemorrhagiae } \\
\text { Autumnalis } \\
\text { Australis }\end{array}$ & 100 & Urbana & $\begin{array}{l}\text { (Romero } \\
\text { et al., } \\
2009 \text { ) }\end{array}$ \\
\hline Tolima & MAT & 20.2 & $\begin{array}{l}\text { Grippotyphosa } \\
\text { Pomona }\end{array}$ & 900 & Urbana & $\begin{array}{l}\text { (Romero y } \\
\text { Sánchez, } \\
\text { 2009) }\end{array}$ \\
\hline Tolima & MAT & 21.4 & $\begin{array}{l}\text { Pomona } \\
\text { Grippotyphosa }\end{array}$ & 850 & Urbana & $\begin{array}{l}\text { (Romero } \\
\text { et al., } \\
2010)\end{array}$ \\
\hline Córdoba & MAT & 12.0 & $\begin{array}{l}\text { Canicola } \\
\text { Icterohaemorrhagiae } \\
\text { Grippotyphosa }\end{array}$ & 200 & Rural & $\begin{array}{l}\text { (Sánchez } \\
\text { et al., } \\
2010)\end{array}$ \\
\hline Boyacá & MAT & 67.2 & $\begin{array}{l}\text { Canicola } \\
\text { Icterohaemorrhagiae } \\
\text { Hardjo } \\
\text { Sejröe } \\
\text { Pomona }\end{array}$ & 61 & $\begin{array}{l}\text { Urbana } \\
\text { Barrios } \\
\text { marginales }\end{array}$ & $\begin{array}{l}\text { (Bermúdez } \\
\text { et al., } \\
\text { 2010) }\end{array}$ \\
\hline Cundinamarca & $\begin{array}{l}\text { Dot- } \\
\text { ELISA } \\
\text { MAT }\end{array}$ & 33.3 & $\begin{array}{l}\text { Canicola } \\
\text { Icterohaemorrhagiae } \\
\text { Grippotyphosa }\end{array}$ & 30 & Urbana & $\begin{array}{l}\text { (Medrano } \\
\text { et al., } \\
2011 \text { ) }\end{array}$ \\
\hline Córdoba & MAT & 47.1 & $\begin{array}{l}\text { Gryppotyphosa } \\
\text { Icterohaemorrhagiae } \\
\text { Pomona }\end{array}$ & 70 & Rural & $\begin{array}{l}\text { (Álvarez et } \\
\text { al., 2011) }\end{array}$ \\
\hline Atlántico & $\begin{array}{l}\text { PCR } \\
\text { MAT }\end{array}$ & 22.9 & $\begin{array}{l}\text { Fainei } \\
\text { Tarassovi } \\
\text { Louisiana }\end{array}$ & 83 & Urbano & $\begin{array}{l}\text { (Romero- } \\
\text { Vivas et } \\
\text { al., 2013) }\end{array}$ \\
\hline
\end{tabular}

Los estudios serológicos de la leptospirosis canina en Colombia (Cuadro 4) han detectado serovares y serogrupos emergentes en la epidemiología de la enfermedad en los últimos años, diferentes a Canicola e Icterohaemorrhagiae, aspecto que se obser- vó en esta investigación. Estos resultados concuerdan con los hallados en otras regiones geográficas como Brasil, Nigeria, Estados Unidos, Canadá y Europa (Okewole y Ayoola, 2009; Oliveira-Lavinsky et al., 2012; Lee et al., 2014; Klaasen y Adler, 2015). Estos 
cambios pueden estar influenciados por nuevos parámetros de transmisión de la infección que incluyen el contacto con transmisores potenciales silvestres y domésticos (Lee et al., 2014), las condiciones sociodemográficas deficientes, la contaminación del ambiente peri-doméstico, la habilidad de los caninos de vagar libremente en un espacio limitado y la densidad de esta especie en las viviendas (Lelu et al., 2015). En esta investigación, no tener más de un perro en la vivienda fue un factor protector que disminuyó la probabilidad de seroreactividad a las leptospiras.

En este estudio, el serogrupo Gryppotyphosa fue el más frecuente en los sueros analizados, coincidente con resultados hallados en los departamentos del Valle del Cauca, Boyacá, Tolima, Córdoba y Cundinamarca (Cuadro 4). Se requiere realizar estudios complementarios para tipificar las leptospiras aisladas de tejidos caninos, así como evaluar la eficacia de la vacuna bivalente utilizada para el control de la enfermedad; la cual incluye los serovares Canicola e Icterohaemorrhagiae. Es importante indicar que en Europa y Estados Unidos se emplean vacunas trivalentes y tetravalentes que incluyen cepas de los serovares Gryppotyphosa y Australis y/o Bratislava, vinculadas con las formas latentes de la enfermedad (Klaasen y Adler, 2015). No obstante, existen limitaciones para seleccionar las mejores vacunas por la dificultad de identificar los serovares locales más importantes, la ineficacia detectada en los biológicos que incluyen un amplio rango de estos y la inmunidad parcial que confieren (Adler y de la Peña Moctezuma, 2010).

En el Valle de Sibundoy, los caninos evaluados presentaron una mayor probabilidad de ser seropositivos a la leptospirosis a medida que aumentó su edad. Similares hallazgos se han descrito en animales atendidos en hospitales veterinarios de Estados Unidos en las últimas tres décadas (Lee et al., 2009), pero divergentes a los hallados en Bahía (Brasil) en población urbana (Oliveira-Lavinsky, 2012). El menor riesgo de contraer la infección de los caninos con edades inferiores a un año puede estar relacionado con una baja o controlada actividad al aire libre, en comparación con otros grupos de mayor edad, o por la inmunidad pasiva conferida a los cachorros vía materna (Lee et al., 2004). No obstante, otros autores han encontrado que los cachorros presentan mayor susceptibilidad a la enfermedad y una menor cobertura de vacunación (Ward y Kelman, 2011).

Un aspecto que llama la atención en el área de estudio es la producción de cuyes (Cavia porcellus), especie que se utiliza como el modelo experimental de elección para el estudio de la leptospirosis aguda y para dilucidar la patogénesis de la infección, la cual es similar a la presentada en la leptospirosis humana de curso grave (Zhang et al., 2012). Dada la alta susceptibilidad de los cuyes a las leptospiras y a la posibilidad de transmisión de la enfermedad a los humanos y otras especies animales, es necesario desarrollar investigaciones complementarias para identificar su importancia en la cadena epidemiológica de la enfermedad.

La alta seroprevalencia de leptospirosis canina observada en este estudio es una evidencia de la transmisión activa de la infección, que puede ser un potencial problema de salud pública en el Valle de Sibundoy. La edad, la falta de tratamiento de las aguas servidas y la densidad de los caninos en la vivienda se relacionaron con la seroreactividad a las leptospiras. Los caninos en entornos suburbanos o rurales tienen un mayor riesgo de adquirir la leptospirosis, debido a la mayor probabilidad de contacto con los hábitats de la vida silvestre. Las autoridades de salud pública deben ser conscientes de los cambios en la dinámica de la transmisión, los serovares circulantes endémicos y de la necesidad del fortalecimiento de la vigilancia epidemiológica de la enfermedad en Colombia, así como el desarrollo de estudios en otras especies animales y en la población humana a riesgo. 


\section{Literatura Citada}

1. Adler B, de la Peña Moctezuma A. 2010. Leptospira and leptospirosis. Vet Microbiol 40: 287-296. doi: 10.1016/ j.vetmic.2009.03.012

2. Álvarez L, Calderón A, Rodríguez V, Arrieta G. 2011. Seroprevalencia de leptospirosis canina en una comunidad rural del municipio de Ciénaga de Oro, Córdoba (Colombia). Rev Udcaactual Divulg Cient 2: 75-81.

3. André-Fontaine G. 2006. Canine leptospirosis - Do we have a problem? Vet Microbiol 117: 19-24. doi: 10.1016/ j.vetmic.2006.04.005

4. Astudillo-Hernández M, GonzálezRodríguez, A, Batista-Santiesteban N, Mirabal-Sosa M, MenéndezHernández J. 2009. Estudio seroepidemiológico de la leptospirosis humana en el departamento del Valle del Cauca, Colombia. Rev Cubana Med Trop 61(2): 1-10.

5. Bermúdez S, Pulido M, Andrade R. 2010. Seroprevalencia de Leptospira spp en caninos y humanos de tres barrios de Tunja, Colombia. Rev MVZ Córdoba 3: 2185-2193.

6. Klaasen HLBM, Adler B. 2015. Recent advances in canine leptospirosis: focus on vaccine development. Vet Med Res Rep 6: 245-260. doi: 10.2147/ VMRR.S59521

7. Lee LHS, Guptill L, Johnson AJ, Moore GE. 2014. Signalment changes in canine leptospirosis between 1970 and 2009. J Vet Intern Med 28: 294-299. doi: 10.1111 /jvim. 12273

8. Lelu M, Muñoz-Zanzi C, Higgins B, Galloway R. 2015. Seroepidemiology of leptospirosis in dogs from rural and slum communities of Los Rios Region, Chile. BMC Vet Res 11: 31-38. doi: 10.1186/ s12917-015-0341-9

9. Levett $P$. 2004. Leptospirosis: a forgotten zoonosis? Clin Appl Immunol Rev 4: 435-448. doi: 10.1016/j.cair.2004.08.001
10. Medrano C, Díaz CA, Dalmau EA. 2011. Diagnóstico de leptospirosis canina por medio de las técnicas DotELISA y MAT en perros con enfermedad renal en Bogotá. Rev Med Vet 21: 133-145. doi: 10.19052/mv.568

11. Okewole EA, Ayoola MO. 2009. Seroprevalence of leptospiral serovars other than Canicola and Icterohaemorrhagiae in dogs in the Southwestern Nigeria. Vet Arhiv 79: 87-96.

12. Oliveira-Lavinsky M, Said RA, Strenzel GM, Langoni H. 2012. Seroprevalence of anti-Leptospira spp antibodies in dogs in Bahia, Brazil. Prev Vet Med 106: 79-84. doi: 10.1016/ j.prevetmed.2012.03.015

13. Ortiz J, Miranda J, Ortiz L, Navarro Y, Mattar S. 2015. Seroprevalencia de Rickettsia sp en indígenas Wayuü de la Guajira y Kankuamos del Cesar, Colombia. Infectio 19: 18-23. doi: 10.1016/ j.infect.2014.11.003

14. Rodríguez AL, Ferro BE, Varona MV1, Santafé M. 2004. Evidencia de exposición a Leptospira en perros callejeros de Cali. Biomédica 24: 291-295. doi: 10.7705/biomedica.v24i3.1275

15. Romero MH, Astudillo M, Quintero ME. 2009. Seroprevalencia y serotipificación de leptospirosis canina en el municipio de Buenaventura (Valle del Cauca). Biosalud 8: 71-76.

16. Romero MH, Astudillo M, Sánchez J, González L, Varela N. 2012. Tiìtulos de anticuerpos contra Leptospira sp en primates del zooloìgico Matecaña, Pereira, Colombia. Rev MVZ Coìrdoba 17: 3224-3230

17. Romero MH, Sánchez J. 2009. Seroprevalencia de la leptospirosis canina de tres municipios del departamento del Tolima-Colombia. Rev MVZ Córdoba 2: 1684-1689.

18. Romero MH, Sánchez JA, Hayek LC. 2010. Prevalencia de anticuerpos contra Leptospira en población urbana humana y canina del Departamento del 
Tolima. Rev Salud Pública 12: 268-275. doi: 10.1590/S0124-00642010000200010

19. Romero-Vivas CME, Cuello-Pérez M, Agudelo-Flórez P, Thiry D, Levett PN, Falconar AK. 2013. Cross-sectional study of Leptospira seroprevalence in humans, rats, mice, and dogs in a main tropical sea-port city. Am J Trop Med Hyg 88: 178-183. doi: 10.4269/ ajtmh.2012.12-0232

20. Sánchez AE, Ballut JC, Calderón A, Rodríguez VC. 2010. Leptospirosis: enfermedad endémica en caninos de áreas rurales de Montería (Córdoba). Orinoquia 14: 160-167.

21. [SIVIGILA] Subdirección de prevención Vigilancia y control en Salud Pública. 2014. Protocolo de Vigilancia en salud Pública. SIVIGILA. 63 p. [Internet]. Disponible en: https:// www.minsalud.gov.co/salud/Paginas/ SIVIGILA.aspx
22. Ward MP, Kelman M. 2011. Companion animal disease surveillance: a new solution to an old problem? Spat Spatiotemporal Epidemiol 2: 147-157. doi: 10.1016/j.sste.2011.07.009

23. Ward MP. 2002. Clustering of reported cases of leptospirosis among dogs in the United States and Canada. Prev Vet Med 56: 215-226. doi: 10.1016/S01675877(02)00160-5

24. Yusti D, Arboleda M, Agudelo-Flórez P. 2013. Factores de riesgo sociales y ambientales relacionados con casos de leptospirosis de manejo ambulatorio y hospitalario, Turbo, Colombia. Biomédica 33: 117-129. doi: 10.7705/biomedica.v33i0.1457

25. Zhang Y, Lou XL, Yang HL, Guo XK, Zhang XY, He P, Jiang XCH. 2012. Establishment of a leptospirosis model in guinea pigs using an epicutaneous inoculations route. BMC Infectio Dis 12: 20-30. doi: 10.1186/1471-2334-12-20 